\title{
Dynamic IEEE 802.11: Design, Modeling and Performance Evaluation
}

\author{
Federico Calì, Marco Conti, and Enrico Gregori \\ CNUCE, Institute of National Research Council \\ Via Alfieri 1 - 56127 Pisa - Italy \\ $\{$ Marco.Conti, Enrico.Gregori\}@cnuce.cnr.it
}

\begin{abstract}
In Wireless LANs (WLANs) the medium access control (MAC) protocol is the main element that determines the efficiency of sharing the limited communication bandwidth of the wireless channel. Previous papers have shown that an appropriate tuning of the backoff algorithm can drive the IEEE 802.11 protocol close to its theoretical limits. In this work we analytically study the performance of the IEEE 802.11 protocol with a dynamically tuned backoff. Specifically, we investigate the sensitiveness of the dynamically tuned backoff algorithm to some network configuration and traffic parameters. Our results indicate that, under stationary traffic conditions, the capacity of the enhanced protocol approaches the theoretical upper bound value in all the configurations analyzed. Furthermore, we also show that the algorithm quickly re-tunes the backoff window size when the network traffic conditions change thus guaranteeing, even with non-stationary traffic conditions, a capacity that is very close to the optimal value. Robustness of the protocol to error conditions is also evaluated. In the paper it is shown the protocol correctly reacts to error conditions.
\end{abstract}

\section{Introduction}

For decades Ethernet has been the predominant network technology for supporting distributed computing. In recent years the proliferation of portable and laptop computers has led to LAN technology being required to support wireless connectivity ([6], [9]). In addition to providing for computer mobility, Wireless LANs (WLANs) are easier to install and save the cost of cabling. The success of WLANs is connected to the development of networking products that can provide wireless network access at a competitive price. A major factor in achieving this goal is the availability of appropriate networking standards. In this paper we focus on the IEEE 802.11 standard for WLANs [8].

The design of wireless LANs has to concentrate more on bandwidth consumption than wired networks. This is because wireless networks deliver much lower

Work carried out under the financial support of CNR, in the framework of the project

"Sistemi radiomobili multimediali nell' evoluzione verso UMTS". 
bandwidths than wired networks, e.g. 1-2 Mbps vs. 10-150 Mbps [11]. Since a WLAN relies on a common transmission medium, the transmissions of the network stations must be coordinated by the Medium Access Control (MAC) protocol. The fraction of channel bandwidth used by successfully transmitted messages gives a good indication of the overheads required by the MAC protocol to perform its coordination task among stations. This fraction is known as the utilization of the channel, and the maximum value it can attain is known as the capacity of the MAC protocol ([10], [5]). Previous works have shown that an appropriate tuning of the IEEE 802.11 backoff algorithm can significantly increase the protocol capacity ([1], [2], [1314]). In [1] the authors propose to tune the backoff window size on the number of active stations, this number being estimated by observing the channel status. Weinmiller et al. [13] outlined a way to modify the backoff distribution to uniformly spread the channel access in a contention window and thus decrease the collision probability. Both studies use simulative analyses to show that significant improvements in protocol capacity can be achieved by modifying the backoff algorithm. In [2] the theoretical upper limit for the IEEE 802.11 protocol capacity was derived. Furthermore, it was shown that with an appropriate tuning of the IEEE 802.11 backoff window size, the protocol capacity can approach the theoretical capacity bound. The results indicate that the optimal backoff window size very much depends on the traffic conditions. Hence the optimal protocol capacity can only be achieved if the backoff window is dynamically tuned at run-time following the evolution of the network configuration (i.e. number of active stations) and load traffic conditions (i.e. average length of transmitted messages). In a previous work we have proposed and analyzed a distributed algorithm to dynamically tune the backoff window-size of the IEEE 802.11 MAC protocol to take into consideration the (dynamically changing) load traffic conditions [Cal 99]. As far as the network configuration is concerned, a fixed network configuration (number $M$ of active stations) is assumed. A performance analysis indicated that, when the number of active stations is not very far from the assumed $M$ value (i.e. in the range between half and the double of the assumed $M$ value) the efficiency of the protocol remains very close to its theoretical bounds. However, by further increasing the distance between $M$ and the real number of active stations the efficiency of the dynamic IEEE 802.11 protocol further degrades thus making it necessary to introduce mechanisms for a dynamic estimation of the $M$ value (see for example [1], [2]). In this work, by exploiting some ideas presented in [2] we extend (and evaluate) the distributed algorithm to dynamically tune the backoff window-size of the IEEE 802.11 MAC protocol to cope also with a dynamically changing number of active stations. Specifically, the proposed algorithm is executed independently by each station that by observing the channel status derives an estimate of both the network and load configurations. Then, by exploiting this information, a station computes the optimal backoff window size for the current configuration. In the following we named Dynamic IEEE 802.11 the IEEE 802.11 MAC protocol extended with such an estimation-based backoff algorithm. By developing a Markovian model of the Dynamic IEEE 802.11 protocol we extensively analyze the properties of the enhanced protocol. Specifically, we study the algorithm's behavior both under steady-state and transient conditions. Furthermore, we analyze the robustness of the 
protocol, that is the protocol ability to cope with errors in the estimations of the network and load configurations. Errors can be induced by the unreliable wireless medium.

The paper is organized as follows. Section 2 presents the IEEE 802.11 MAC protocol and the dynamically tuned backoff algorithm. In Sections 3 and 4 we study the protocol behavior in steady-state and in transient conditions, respectively. Finally in Section 5 we study the protocol robustness.

\section{IEEE 802.11 MAC Protocol}

The IEEE 802.11 MAC layer protocol provides asynchronous, time-bounded, and contention free access control on a variety of physical layers. The basic access method in the IEEE 802.11 MAC protocol is the Distributed Coordination Function (DCF) which is a Carrier Sense Multiple Access with Collision Avoidance (CSMA/CA) MAC protocol [8].

The model used in this paper to evaluate the protocol performance figures does not depend on the technology adopted at the physical layer (e.g. infrared and spread spectrum). However, the physical layer technology determines some network parameter values, e.g. SIFS, DIFS, and backoff slot time. Whenever necessary, we choose the values of these technology-dependent parameters by referring to the frequency-hopping-spread-spectrum technology at a 2 Mbps transmission rate.

\subsection{Theoretical Capacity Limits of the IEEE 802.11 Protocol}

In [2] the efficiency of the IEEE 802.11 standard for wireless LANs was investigated in depth. Specifically, by deriving an analytical formula for the protocol capacity: $i$ ) the theoretical upper bound of the IEEE 802.11 protocol capacity was identified, and ii) it was shown that, depending on the network configuration, the standard may operate very far from the theoretical limits. A summary of these results is reported in Figure 1 that compares, for several network configurations, the IEEE 802.11 capacity with the analytical bounds. These results have been obtained with the configuration parameter values reported in Table 1.

Table 1. WLAN configuration

\begin{tabular}{cc}
\hline Parameter & value \\
\hline SIFS & $28 \mu \mathrm{sec}$ \\
DIFS & $128 \mu \mathrm{sec}$ \\
backoff slot time & $50 \mu \mathrm{sec}$ \\
bit rate & $2 \mathrm{Mbps}$ \\
propagation delay & $1 \mu \mathrm{sec}$ \\
stations & $10,50,100$ \\
CWmin & 8 \\
CWmax & 256 \\
\hline
\end{tabular}


The results show that for almost all configurations the IEEE 802.11 capacity can be improved significantly. As highlighted by the figure, the distance between the IEEE 802.11 and the analytical bound increases with the number, $M$, of active stations. The figure also shows an additional curve tagged as optimal window size. This curve represents the capacity of an IEEE 802.11 protocol but with a single contention window whose size is equal to the optimal value (for that network and traffic configuration) that is computed from the formulas developed for the theoretical upper bound of the capacity [2].

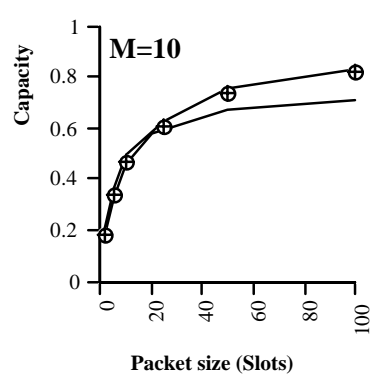

$\oplus \quad$ Optimal window size

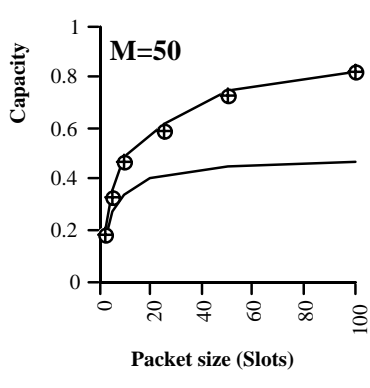

Analytical bound

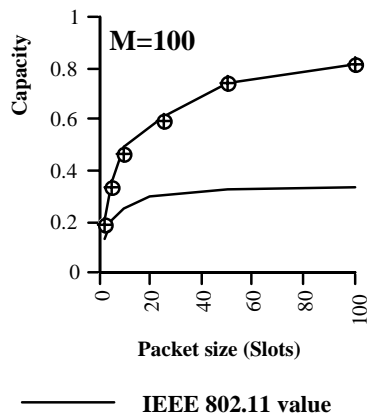

IEEE 802.11 value

Fig. 1. Analytical bounds vs. IEEE 802.11 capacity

\subsection{Dynamic IEEE 802.11}

The results presented in the previous section indicate that the IEEE 802.11 protocol often operates very far from its theoretical limits and that the theoretical capacity limits can be achieved with an adequate tuning of the backoff window size. Based on these observations, in this paper we investigate the Dynamic IEEE 802.11 protocol, i.e. the IEEE 802.11 protocol with an algorithm for the dynamic tuning of the backoff parameters.

The results presented in [2] indicate that, given a network and traffic configuration, there exists an optimal value for the average backoff interval $E[B]$. This optimal value can be easily mapped on the value of the parameter $p$ of a geometric distribution with an average $E[B]$. Hereafter $p_{\min }$ will indicate the $p$ value corresponding to the optimal backoff interval.

The advantage of using a geometrically distributed backoff with respect to the uniformly distributed backoff of the IEEE 802.11 is that by simply changing the $p$ parameter value we can immediately adapt the backoff window size to a change in the network or traffic conditions.

The Dynamic IEEE 802.11 protocol is similar to a $p$-persistent protocol [11]: at the beginning of an empty slot a station transmits (in that slot) with a probability $p$. The differs With a probability 1- $p$ the transmission is deferred. This procedure is repeated whenever an empty slot is detected on the channel. The main differences between and a classical $p$-persistent protocol are: 
- in a classical $p$-persistent protocol the value of the $p$-parameter is constant while in the Dynamic IEEE 802.11 protocol the $p$ value changes depending on the network configuration and load conditions;

- in a classical p-persistent protocol the length of the backoff interval is independent of the status of the channel during the backoff itself, while in the Dynamic IEEE 802.11 protocol, as in the standard IEEE 802.11 protocol, the backoff decreases only when the channel is idle.

The main element of the Dynamic IEEE 802.11 protocol, with respect to the standard one is the algorithm that is in charge to dynamically adjust the $p$-value to the network and load conditions. In [3] it was proposed and evaluated an algorithm that maximizes the protocol capacity by dynamically adapting the $p$-value to the load configuration. However, the $p$-estimation algorithm proposed in [3] assumed that each station knew the number $M$ of the active stations in the network a priori. This is a strong assumption as, in the real network, the number of active stations varies considerably.

Here, we propose and analyze a dynamic backoff algorithm that does not require any a priori knowledge on the network and load conditions. Specifically the algorithm, by observing the channel status, estimates at run-time both the number of active stations in the network and the load configuration. By exploiting this information the algorithm updates the $p$-value. The updates of the $p$-value occur at the end of very virtual transmission time, where a virtual transmission time is the time interval between two consecutive transmission attempts.

Before presenting the procedure used by the Dynamic IEEE 802.11 protocol to estimate the load and network configurations, it is useful to better identify the information required by the protocol to compute the optimal $p$-value.

Optimal $p$-value. In this section we define a criteria to compute the $p$-value to guarantee that the Dynamic IEEE 802.11 protocol achieves the theoretical protocol capacity, i.e. the maximum throughput in the current load and network configuration.

In [2], by exploiting the geometric backoff assumption, it has been shown that the protocol capacity can be expressed as:

$$
\rho_{\max }=\frac{\bar{m}}{E\left[t_{\text {success }}\right]}
$$

where $E\left[t_{\text {success }}\right]$ is the average temporal distance between two consecutive successful transmissions, and $\bar{m}$ is the average message length.

Furthermore, for a given network and load configuration, $E\left[t_{\text {success }}\right]$ is a function of the $p$-value only. Hence, the value of $p$ that minimizes $E\left[t_{\text {success }}\right]$, say $p_{\min }$, guarantees the maximum protocol capacity. Since the exact $p_{\min }$ derivation is expensive from a 
computational standpoint, in [2], it was proposed to approximate $p_{\min }$ with the $p$ value that satisfies the following relationship: 1

$$
E[\text { Coll }]_{]_{\text {Collision }}} \cdot E\left[N_{c}\right]=\left(E\left[N_{c}\right]+1\right) \cdot E[\text { Idle }-p] \cdot t_{\text {slot }}
$$

where $E[\mathrm{Coll}]]_{\text {Collision }}$ is the average collision length, $E\left[N_{c}\right]$ is the average number of collisions between two consecutive successful transmissions, and $E\left[\right.$ Idle $\left.e_{-}\right]$is the average number of consecutive idle slots.

By noting that $E\left[N_{c} \bigvee\left(E\left[N_{c}\right]+1\right)\right.$ is the probability that a collision occurs given a transmission attempt, Equation (1) can be written as:

$$
E[\text { Coll }]_{\text {collision }}=\frac{E[\text { Idle } p] \cdot t_{\text {slot }}}{p_{\text {collision }}} \Rightarrow E[\text { Coll }]=E[\text { Idle } p] \cdot t_{\text {slot }}
$$

where $p_{\text {collision }}=E\left[N_{c}\right]\left(E\left[N_{c}\right]+1\right)$, and Coll is the time the channel is busy due to a collision given that a transmission attempt occurs, also referred to as collision cost. Obviously, Coll is equal to zero if the transmission attempt is successful, otherwise it is equal to the collision length.

Analytical formulas for the quantities of Equation (2) are defined in LEMMA 1 whose proof can be found in [2].

LEMMA 1. Assuming that for each station i) the backoff interval is sampled from a geometric distribution with parameter $p$, and ii) packet lengths are i.i.d. sampled from a geometric distribution with parameter $q$ :

$$
\begin{aligned}
& E\left[N_{c}\right]=\frac{1-(1-p)^{M}}{M p(1-p)^{M-1}}-1 \\
& E[\text { Coll }]_{\text {lcollision }}=\frac{t_{\text {slot }}}{1-\left[(1-p)^{M}+M p(1-p)^{M-1}\right]} . \\
& {\left[\sum_{h=1}^{\infty}\left\{r \cdot\left[\left(1-p q^{h}\right)^{M}-\left(1-p q^{h-1}\right)^{M}\right]\right\} \frac{M p(1-p)^{M-1}}{1-q}\right]}
\end{aligned}
$$

As it clearly, appears, from LEMMA 1, the quantities of Equation (2) can be expressed as a function of $p$. Hence, Equation (2) provides the criteria to identify the $p$-value that maximizes the protocol capacity. Specifically, by observing the channel status, a station after each transmission attempts updates its estimate of the average collision cost, $E[\mathrm{Coll}]$. Hence, if the station has an estimate of the number of active stations, $M$, by exploiting the formula

$$
E[\text { Idle }-p]=\frac{(1-p)^{M}}{1-(1-p)^{M}}
$$

\footnotetext{
${ }^{1}$ A similar approximation of the optimal point was proposed in [7] for an Aloha CSMA protocol.
} 
it can compute the value of $p$ that satisfies the optimal criteria defined by Equation (2).

To summarize, a station to implement the dynamic backoff algorithm needs the knowledge of $M$ or at least of an estimate of it, say Me, $E[$ Coll $]$ and $E\left[I d l e_{-} p\right]$. In the next section we show how a station can obtain these information by manipulating the information related to the channel status.

Run-time estimates. As stated in the previous section a station modifies the $p$ value that it uses for the backoff algorithm at the end of each virtual transmission interval (i.e. after each transmission attempt).

To better clarify the operations performed by a station let us refer to Figure 2. Specifically, the figure represents a station behavior during the $(n+1)$ th virtual transmission interval by assuming that at the beginning of that interval, i.e. the end of the $n$-th virtual transmission interval, it has the following information:

- $\quad p_{n}$ is the optimal value of $p$;

- $\quad M e_{n}$ is the estimated number of active stations;

- $\quad E\left[I d l e_{-} p\right]_{n}$ is the average number of consecutive empty slots;

- $\quad E[\mathrm{Coll}]_{n}$ is the average collision cost.

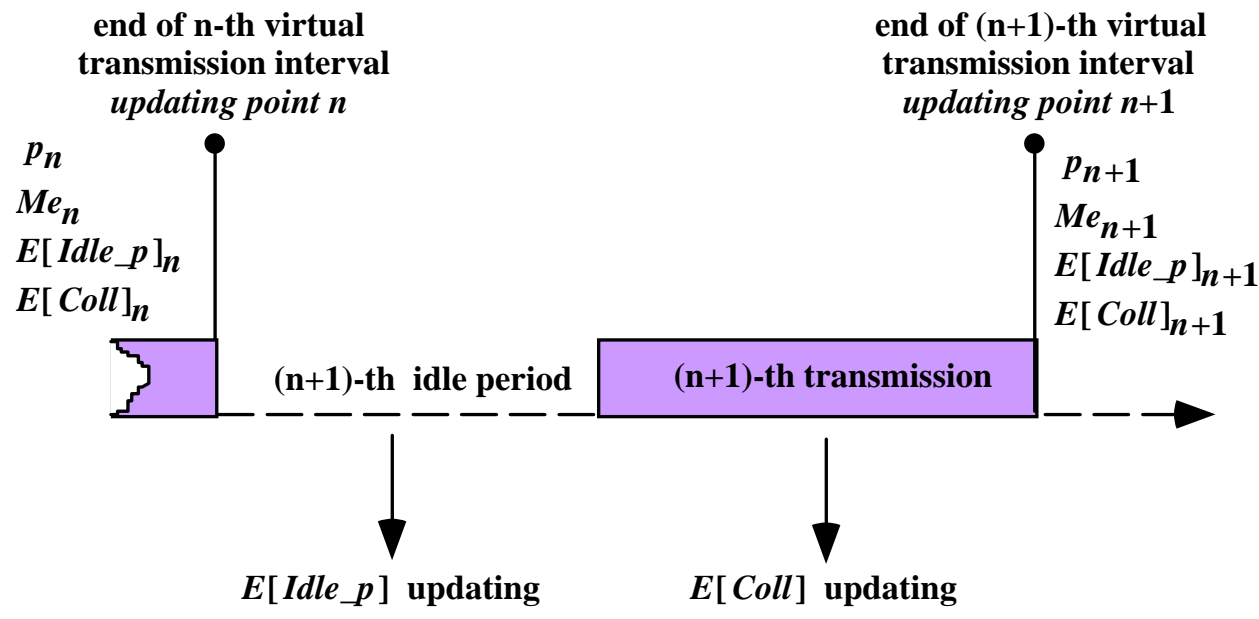

Fig. 2. Estimates updating

Each station by using the carrier sensing mechanism can observe the channel status 2 and measure the length of both the last idle period and the last transmission attempt.

${ }^{2}$ In a CSMA protocol a station observes all the channel busy periods. A busy period is assumed to be a collision if an ACK does not immediately follow. 
From this two values, the algorithm, by exploiting a moving averaging window, approximates the average idle period length and the average collision length:

$$
\begin{aligned}
& E\left[\text { Idle } e_{-}\right]_{n+1}=\alpha \cdot E[\text { Idle } \\
& -p]_{n}+(1-\alpha) \cdot I d_{n+1}
\end{aligned}
$$

where $E\left[\text { Idle } e_{-} p\right]_{n+1}$ and $E[\text { Coll }]_{n+1}$ are the approximations of $E\left[\right.$ Idle $\left.\_p\right]$ and $E[C o l l]$ respectively at the end of the $(n+1)$ th transmission attempt, $I d_{n+1}$ is the length of the $(n+1)$ th idle period, $T a_{n+1}$ is the length of the $(n+1)$ th transmission attempt and $\alpha$ is a smoothing factor. The use of a smoothing factor, $\alpha$, is widespread in the networking environment to obtain reliable estimates from the network estimates by avoiding harmful fluctuations e.g., RTT estimation in TCP [12].

It is worth noting that $E\left[I d l e_{-} p\right]_{n+1}$ is estimated by observing the channel status, hence its value is function of the $p$-value used by the stations and the real number $M$ of active stations. Hence, from the knowledge of $E\left[\text { Idle } e_{-} p\right]_{n+1}$ and the $p$-value a station can derive an estimate of the number of active stations, $M e_{n+1}$. Specifically, at the end of the $(n+1)$ th virtual transmission interval each station computes an estimate of $M$, say $M_{\text {comp }}$, by exploiting $E\left[\text { Idle } e_{-} p\right]_{n+1}$ and the formula defined in Lemma 1 that expresses $E\left[I d l e_{-} p\right]$ as a function of $p$ and $M$ :

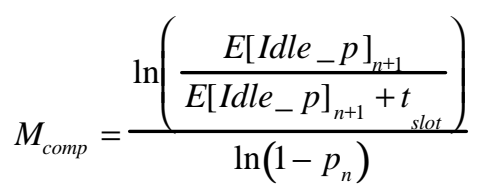

Finally, from (4), the new estimate of the number of active station $M e_{n+1}$ is computed by:

$$
M e_{n+1}=\alpha \cdot M_{\text {comp }}+(1-\alpha) \cdot M e_{n}
$$

The updated estimate of the number of active stations $M e_{n+1}$ is then used together with $E[\text { Coll }]_{n+1}$ (see (3)) to compute the value of $p$ that is optimal (i.e. maximizes the protocol capacity) for the new network and load conditions. Specifically, according to Equation (2), the optimal $p$-value should guarantee a balance between the average collision cost and the average idle-period length. Each station, by exploiting Lemma 1 , expresses the average idle-period length as a function of $p$ and of the number of active stations (note that a station does not have the knowledge of this number but has an estimate of it, $\left.M e_{n+1}\right)$. Then, by using its estimate of $E[\mathrm{Coll}]_{n+1}$ computes the new optimal value of $p, p_{n+1}$, from the following formula:

$$
p_{n+1}=1-M e_{(n+1)} \sqrt{\frac{E[\text { Coll }]_{n+1}}{E[\text { Coll }]_{n+1}+t}} .
$$


The feasible range of $p$ values has 1 as its upper bound (a station can use all the channel bandwidth when it is alone). The lower bound is set to the optimal $p$ value for the maximum number of station allowed in the network (e.g. 100 or 500).

In [2] it was shown that, in a IEEE 802.11 WLAN, if each station tunes its backoff algorithm to the optimal $p$-value for the current network and load configuration, the MAC protocol capacity is very close to its theoretical bound. In this work we have presented an algorithm which first estimates the current network and load configuration and then from this knowledge it tunes its backoff algorithm. Hence, in this case the knowledge a station has about the network and load configuration is not exact but depends on an estimation process. In the next sections, by exploiting the Markovian model developed in [4] we study the behavior of the dynamic backofftuning algorithm. Specifically, we investigate the protocol performance both in steady-state and transient conditions. In addition, we investigate the robustness of the protocol to possible errors during the estimation process. These errors can occur as the estimation process is based on the carrier sensing of the channel, and it is well known that wireless channels are unreliable.

\section{Dynamic IEEE 802.11: Protocol Behavior in Steady State Conditions}

Table 2 compares, for two network $(M=10,20)$ and traffic $(q=0.5,0.99)$ configurations, the protocol capacity values obtained with our analytical model with the theoretical upper bounds derived in [2] $3^{3}$ Furthermore, we analyze the impact of the $\alpha$ smoothing value $(\alpha=0.5,0.90,0.99)$ on the steady-state behavior of the Dynamic IEEE 802.11 protocol.

Table 2. Dynamic IEEE 802.11 Protocol capacity $(M=10)$

\begin{tabular}{ccccccc}
\hline & \multicolumn{2}{c}{$\bar{m}=100$ slots $(q=0.99)$} & \multicolumn{3}{c}{$\bar{m}=2$} & \multicolumn{2}{c}{ slots $(q=0.50)$} \\
\hline$\alpha=0.50$ & 13.09 & 0.00831 & 0.8018 & 13.36 & 0.0427 & 0.2004 \\
$\alpha=0.90$ & 10.33 & 0.01058 & 0.8220 & 9.17 & 0.0685 & 0.2009 \\
$\alpha=0.99$ & 9.78 & 0.01192 & 0.8237 & 10.16 & 0.0559 & 0.2081 \\
IEEE & & & 0.7135 & & & 0.1818 \\
802.11 & & & & & & \\
ideal & 10 & 0.01150 & 0.8257 & 10 & 0.0525 & 0.2088 \\
values & & & & & & \\
\hline
\end{tabular}

3 The analytical results were obtained with $\alpha=0.9$, and 20 states in the set $I$. 
Table 3. Dynamic IEEE 802.11 Protocol capacity $(M=20)$

\begin{tabular}{|c|c|c|c|c|c|c|}
\hline \multirow[b]{2}{*}{$\alpha=0.50$} & \multicolumn{3}{|c|}{$\bar{m}=100$ slots $(q=0.99)$} & \multicolumn{3}{|c|}{$\bar{m}=2$ slots $(q=0.50)$} \\
\hline & 27.14 & 0.00388 & 0.7972 & 27.41 & 0.0210 & 0.1990 \\
\hline$\alpha=0.90$ & 18.57 & 0.00517 & 0.8126 & 17.58 & 0.0282 & 0.1985 \\
\hline $\begin{array}{c}\alpha=0.99 \\
\text { IEEE }\end{array}$ & 19.00 & 0.00603 & 0.8214 & 19.98 & 0.0279 & 0.2057 \\
\hline $\begin{array}{c}802.11 \\
\text { ideal }\end{array}$ & & & 0.6113 & & & 0.1789 \\
\hline values & 20 & 0.00572 & 0.8223 & 20 & 0.0279 & 0.2060 \\
\hline
\end{tabular}

The results show that the dynamic tuning algorithm is very effective for the network and traffic configuration analyzed. As shown by the analytical results presented in Tables 2 and 3, the capacity of a WLAN implementing the dynamic-backoff tuning algorithm is always very close to the theoretical capacity upper bound (see the idealvalue line in the two tables). Furthermore, the tables show, as expected, the impact of the $\alpha$ smoothing value. As we are investigating the protocol behavior in stationary conditions, with the increase of the $\alpha$ values the statistical fluctuations of the quantities estimated by observing the status of the channel becomes less relevant and thus the idle-period and collision-cost estimates are always very close to their steadystate average values. Our results indicate that $\alpha=0.50$ is not appropriate and $\alpha=0.99$ is the best choice for a system operating in stationary conditions. However, $\alpha=0.90$ provides statistics that are quite close to the ideal values as well, and we can expect that $\alpha=0.90$ is more appropriate when the load and/or network conditions changes because it potentially reduces the length of transient phases.

\section{Dynamic IEEE 802.11: Protocol Behavior in Transient Conditions}

In this section we analyze the protocol promptness to re-tuning the backoff parameters when the network state sharply changes. Results presented in Table 4 are obtained as follows: the network is assumed to be in steady state with 2 active nodes (20 active nodes). This means that at time 0 we assume that the estimated $M$ is 2 (20) and the $p$ value used for the backoff algorithm is the theoretically-optimal $p$ value for $M=2(M=20)$. At time $0^{+}$the number of active nodes becomes 10 . Exploiting our analytical model we evaluate the average first passage time to the new steady state, i.e., the time to update the $M$ estimate to 10 . This is done through the standard procedure for the computation of the first-passage-time distribution in a Markov chain. At the step 0 of the procedure, the system is with probability one in the optimal state for $M=2$. 
Table 4. Average first-passage time to the new steady state

\begin{tabular}{ccc}
\hline starting state & $M=2, \operatorname{popt}(2)$ & $\mathrm{M}=20, \mathrm{p}_{\mathrm{opt}}(20)$ \\
\hline$\alpha=0.5$ & $9.2 \mathrm{sec}$. & $6.8 \mathrm{sec}$. \\
$\alpha=0.9$ & $40 \mathrm{sec}$. & $12 \mathrm{sec}$. \\
$\alpha=0.95$ & $231 \mathrm{sec}$. & $84 \mathrm{sec}$. \\
$\alpha=0.99$ & $4000 \mathrm{sec}$. & $2090 \mathrm{sec}$. \\
\hline
\end{tabular}

Table 4 presents the average first passage time for various $\alpha$ values. These first passage times remain quite short for smoothing factor $\alpha$ up to 0.9. Increasing further the $\alpha$ value makes the transient phase significantly longer. The minimum transient is obviously obtained with $\alpha=.5$, but as shown in the steady-state analysis (see Section 4), $\alpha=.5$ makes our dynamic algorithm too tied to the fluctuations of the network estimates, thus reducing the protocol capacity.

Results presented in Table 4 and in Section 4 indicate that $\alpha=.9$ is a good compromise between precision and promptness.

The difference between the average first-passage time from $M=2$ to $M=10$, and from $M=20$ to $M=10$ can be explained by noting that in the former case the estimated $M$ has to increase five times, while in the latter case it is reduced to its half.

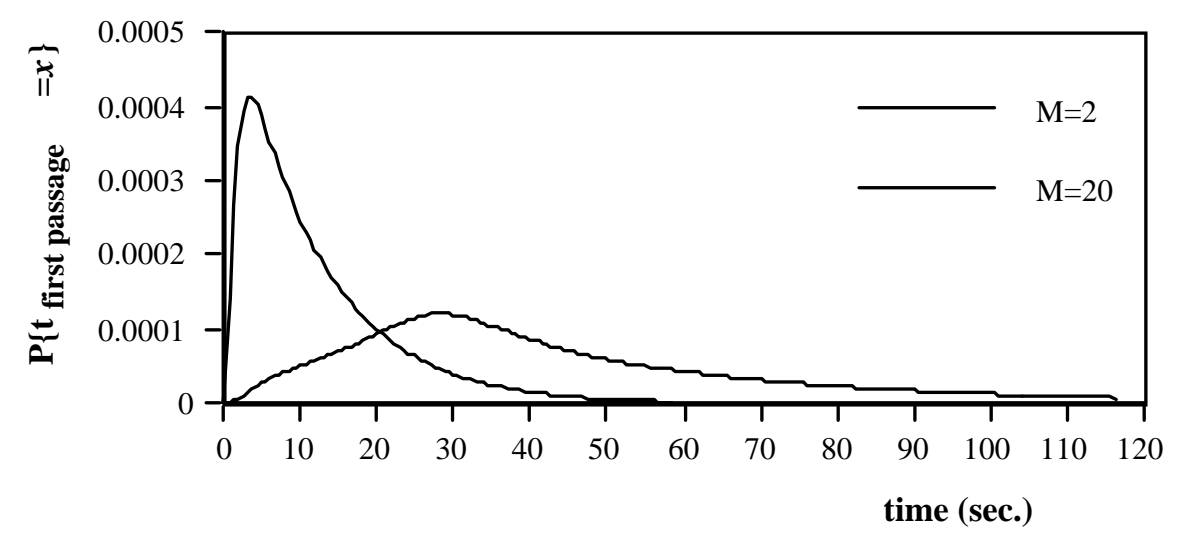

Fig. 3. First passage time distribution

To better analyze the first-passage-time statistics, in Figure 3 we plot the steady-state distribution of the first passage time for $\alpha=.9$. The figure indicates that transient intervals are, in the worst case, about one or two minutes. These values are not critical also because it is very unlikely that such a sharp change in the traffic profile occurs in a realistic scenario. 


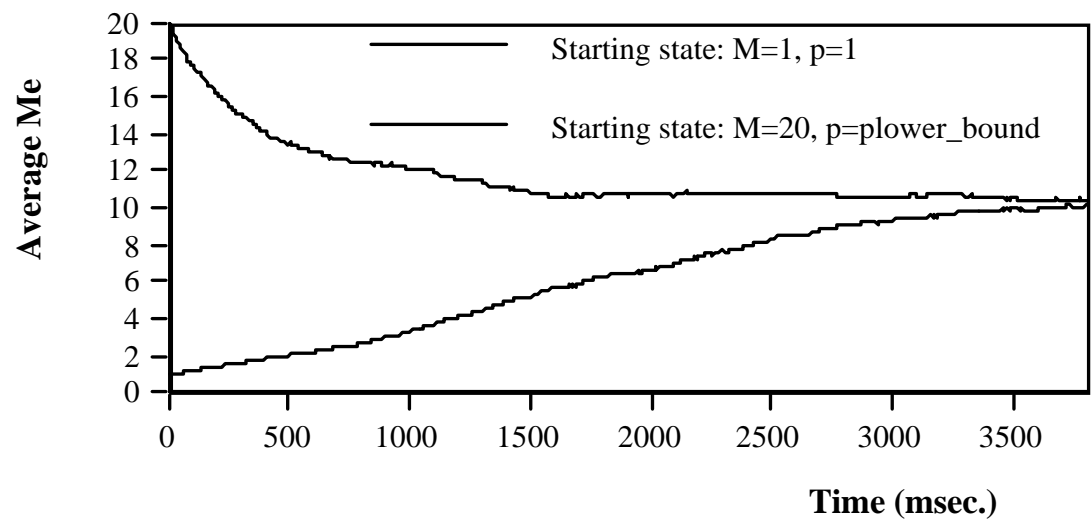

Fig. 4. System behavior when the staring state is wrong

\section{Dynamic IEEE 802.11: Protocol Robustness to Estimation Errors}

In this subsection we discuss the robustness of the protocol. We assume that due to some errors in the estimation phase or biasing induced by the hidden-station phenomenon all the $M$ active stations assume $M e=1$ and thus $p=1$. Thus a collision will immediately occur and, as a result of the collision, the $p$ parameter value used in the next backoff is much smaller (0.0853). From this time onward the evolution is probabilistic. Figure 4 plots the average $M e$ estimate. This estimate is computed using standard transient-analysis method for markovian systems. The Markov chain used for this analysis corresponds to a 10 active stations scenario, i.e. $(M=10)$. The figure indicates that the system correctly reacts to the wrong estimate and, in few seconds, the estimates for $M$ and then for $p$ become the optimal ones.

On the other hand, wrongly assuming a highly-congested network $(M=20$ and $p=$ minimum value) when $M=10$ is less critical. The evolution of the $M e$ is probabilistic and thus its study requires a transient Markov chain analysis. The average $M e$ plotted in Figure 4 indicates that the system correctly reacts to the wrong estimate in this case too and after a few seconds the correct $M e$ value is reached.

\section{References}

[1] G.Bianchi, L.Fratta, M. Oliveri, "Performance Evaluation and Enhancement of the CSMA/CA MAC Protocol for 802.11 Wireless LANs", Proceedings of PIMRC 1996, October 1996, Taipei, Taiwan, pp. 392-396.

[2] F.Calì, M. Conti, E. Gregori, "IEEE 802.11 Wireless LAN: Capacity Analysis and Protocol Enhancement", Proc. INFOCOM 98, San Francisco, March 1998.

[3] F.Calì, M. Conti, E. Gregori, "Performance Modeling of an Enhanced IEEE 802.11 protocol", Proc. IFIP ATM 99, June 1999, Antwerp, Belgium,. 
[4] F.Calì, M. Conti, E. Gregori, "IEEE 802.11 protocol: design and performance evaluation of an adaptive backoff mechanism" CNUCE report, September 1999.

[5] M. Conti, E. Gregori, L. Lenzini, "Metropolitan Area Networks" Springer Verlag, November 1997.

[6] G.H. Forman, J. Zahorjan, “ The challenges of mobile computing”, IEEE Computer, April 94, pp.38-47.

[7] R.G. Gallager, "A perspective on multiaccess channels", IEEE Trans on Information Theory, Vol.31, 1985, pp. 124-142.

[8] IEEE standard for Wireless LAN- Medium Access Control and Physical Layer Specification, P802.11, November 1997.

[9] T. Imielinsky, B.R. Badrinath, "Mobile Computing: Solutions and Challenges in Data Management", Communications of ACM , Oct. 94.

[10] J.F. Kurose, M. Schwartz, Y. Yemini, "Multiple access protocols and time constraint communications", ACM computing Surveys, Vol. 16, pp.43-70.

[11] W. Stallings, "Local \& Metropolitan Area Networks", Prentice Hall, 1996.

[12] W.R.Stevens, "TCP/IP Illustrated, Volume 1: The Protocols", Addison-Wesley, Reading, MA, 1994

[13] J. Weinmiller, H. Woesner, JP Ebert, A. Wolisz, " Analyzing and Tuning the Distributed Coordination Function in the IEEE 802.11 DFWMAC Draft Standard", Proceedings of MASCOT '96, February 1996, San Jose, California.

[14] J. Weinmiller, M. Schläger, A. Festag, A. Wolisz, " Performance Study of Access control in Wireless LANs-IEEE 802.11 DFWMAC and ETSI RES 10 HIPERLAN", Mobile Networks and Applications, Vol. 2, 1997, pp.55-67. 